DOI: https://doi.org/10.34883/PI.2021.13.5.010

УДК 616.127-002/.008-079.4

Курлянская Е.К.

Республиканский научно-практический центр «Кардиология», Минск, Беларусь

Kurlianskaya A.

Republican Scientific and Practical Centre "Cardiology", Minsk, Belarus

\title{
Дифференциальная диагностика
} дилатационной и воспалительной

\section{кардиомиопатии}

\author{
Differential Diagnostics of Dilated and Inflammatory \\ Cardiomyopathy
}

Резюме

Цель. Определить критерии дифференциальной диагностики воспалительной (ВКМП) и дилатационной кардиомиопатии (ДКМП).

Материалы и методы. В исследование включены 235 пациентов с впервые выявленной кардиомиопатией (КМП) неишемического генеза. С целью подбора пациентов с ДКМП и ВКМП, сопоставимых по состоянию внутрисердечной гемодинамики и достижения исходной гомогенности групп, применяли метод PSM (Propensity score matching). Классифицирующую способность количественных показателей оценивали с помощью ROC-анализа. Индикатором диагностической ценности показателя служила площадь под ROC-кривой (AUC - area under curve) с нижней границей 95\% ДИ не менее 0,60. Наилучшим порогом отсечения (cut-off) для тестируемой переменной являлось значение с оптимальным балансом чувствительности и специфичности. Величина критического уровня значимости (р) для всех критериев и тестов принималась равной 0,050.

Результаты. В целом по выборке пациентов с неишемической кардиомиопатией получены статистически значимые взаимосвязи показателей, ассоциированных с миокардиальным фиброзом. Объем экстрацеллюлярного матрикса миокарда ЛЖ умеренно коррелировал с фиброзом в биоптатах миокарда $\left(r_{s}=0,40, p=0,019\right)$ и вольтажем униполярного электрода $\left(r_{s}=-0,43, p=0,009\right)$. В свою очередь вольтаж униполярного электрода заметно коррелировал с гистологическим параметром фиброза $\left(r_{s}=-0,54, p<0,001\right)$. Следует отметить, что в гистологической картине кардиомиопатии наличие выраженного интерстициального фиброза, как правило, с развитием заместительного склероза, является основной отличительной чертой ВКМП. При этом, несмотря на отсутствие активного воспаления, для постмиокардитической кардиомиопатии характерно наличие рассеянных круглоклеточных воспалительных инфильтратов. Важно отметить, что в отличие от активного миокардита воспалительные инфильтраты не ассоциированы с повреждением миоцитов.

Выводы. Характерными особенностями впервые выявленной ВКМП являются более высокие, чем при ДКМП, количественные показатели фиброза миокарда Лж (сегментарные значения времени преконтрастной релаксации $(\mathrm{p}=0,002)$ и объема экстрацеллюлярного матрикca $(p=0,006))$ на фоне более низких показателей толщины миокарда ЛЖ в систолу $(p=0,039)$ и диастолу ( $p=0,043)$. Дополнительными диагностическими критериями ВКМП являются время преконтрастной релаксации $\geq 1031$ мс и объем экстрацеллюлярного матрикса $\geq 28 \%$ не менее 
чем в 8 сегментах миокарда ЛЖ. Дополнительные критерии диагностики ДКМП - время преконтрастной релаксации < 1031 мс и объем экстрацеллюлярного матрикса $<28 \%$.

Ключевые слова: дилатационная кардиомиопатия, воспалительная кардиомиопатия, магнитно-резонансная томография.

\section{Abstract}

Purpose. To determine the differential diagnosis criteria for inflammatory (ICM) and dilated cardiomyopathy (DCM).

Materials and methods. The study involved 235 patients with newly diagnosed non-ischemic cardiomyopathy. Propensity score matching (PSM) was applied to select patients with ICM and DCM with comparable intracardiac hemodynamics and to make our groups initially homogenous. The classifying ability of quantitative parameters was assessed using ROC analysis. The diagnostic value of the parameters was marked by the area under the ROC curve (AUC - area under the curve) with a lower limit of $95 \% \mathrm{Cl}$ of at least 0.60 . The best cut-off threshold for the variable being tested was considered a value with an optimal balance of sensitivity and specificity. The value of the critical level of significance (p) for all parameters and tests was taken to be 0.050 .

Results. As a result, a statistically significant correlation of parameter values associated with myocardial fibrosis was found in the sample of patients with non-ischemic cardiomyopathy. The volume of the LV myocardium extracellular matrix moderately correlated with fibrosis in myocardial biopsy ( $r s=0.40, p=0.019$ ) and the voltage of the unipolar electrode ( $r s=-0.43, p=0.009$ ). In turn, the voltage of the unipolar electrode significantly correlated with the histological parameter of fibrosis $(r s=-0.54, p<0.001)$. It should be noted that in cardiomyopathy histology, the main distinguishing feature of ICM is the presence of severe interstitial fibrosis, generally with the development of secondary sclerosis. However, despite the absence of active inflammation, postmyocarditis cardiomyopathy is characterized by the presence of multiple round-cell inflammatory infiltrates. It is important to note that, unlike in cases of active myocarditis, inflammatory infiltrates are not associated with myocyte damage.

Conclusions. As a result, we discovered that for the newly diagnosed ICM, it is typical to show higher quantitative parameters of LV myocardial fibrosis than in DCM (segmental values of pre-contrast relaxation time $(p=0.002)$ and extracellular matrix volume $(p=0.006))$ on the background of lower values of LV myocardial thickness in systole $(p=0.039)$ and diastole $(p=0.043)$. Additional diagnostic criteria for ICM are pre-contrast relaxation time $\geq 1031$ ms and extracellular matrix volume $\geq 28 \%$ in at least 8 segments of the LV myocardium. Additional criteria for DCP diagnosis are pre-contrast relaxation time $<1031 \mathrm{~ms}$ and extracellular matrix volume $<28 \%$.

Keywords: dilated cardiomyopathy, inflammatory cardiomyopathy, magnetic resonance imaging.

\section{- ВВЕДЕНИЕ}

Кардиомиопатии являются одной из ведущих причин инвалидизации и смертности как в нашей стране, так и за рубежом. Далеко не единственной этиологической причиной ХСН является ИБС. Все большую социальную значимость приобретает проблема кардиомиопатий некоронарогенной этиологии, поскольку данная патология часто встречается у лиц молодого и трудоспособного возраста. В настоящее время пересмотрены многие патогенетические концепции, усовершенствованы диагностические технологии, появился целый ряд новых методик, которые могут расширить возможности оценки не только механизмов 
формирования, но и выбора наиболее эффективных методов диагностики и лечения неишемических кардиомиопатий [1]. В доступной литературе основная часть работ по лечению $\mathrm{XCH}$ посвящена целой группе кардиомиопатий ишемической и неишемической этиологии без разделения последней на идиопатическую дилатационную кардиомиопатию (ДКМП) и воспалительную кардиомипатию (ВКМП) [2]. В то же время около 2/3 пациентов с кардиомиопатией имеют воспалительную природу развития заболевания, связанную с перенесенным ранее миокардитом [3]. Изменения в миокарде у пациента с ВКМП отличаются от таковых при идиопатической ДКМП, так как связаны с прямым агент-зависимым повреждением миокарда, а также с возникновением аутоиммунных реакций [4]. Разрастание соединительной ткани вследствие перенесенного воспалительного процесса становится причиной нарушения эластичности и сократимости сердечной мышцы. Клиническая картина заболевания мало отличается от других хронических заболеваний сердца. На первый план выходят симптомы сердечной недостаточности, обусловленной нарушением питания миокарда. Международная классификация болезней шифрует ВКМП в отделах статистики по коду «Другие поражения сердца при различных заболеваниях».

На сегодняшний день не существует алгоритмов диагностики ВКМП, обладающих высокой степенью диагностической надежности. Общепринятым методом подтверждения ВКМП является эндомиокардиальная биопсия. Однако это достаточно сложная, дорогостоящая инвазивная процедура, сопряженная с высоким риском для пациента. Для дифференциальной диагностики кардиомиопатий используется магнитнорезонансная томография. Этот высокочувствительный и эффективный метод диагностики является безопасным для пациента. Но в настоящее время отсутствуют четкие диагностические МРТ-критерии, позволяющие дифференцировать изменения миокарда воспалительной этиологии от других неишемических заболеваний сердечной мышцы. Клиническая диагностика ВКМП затруднительна из-за сходства проявлений заболевания с рядом других сердечных патологий.

\section{— ЦЕЛЬ ИССЛЕДОВАНИЯ}

Определить критерии дифференциальной диагностики воспалительной и дилатационной кардиомиопатии.

\section{- МАТЕРИАЛЫ И МЕТОДЫ}

В исследование включено 235 пациентов с впервые выявленной кардиомиопатией (КМП) неишемического генеза. Средний возраст пациентов $-45,8 \pm 3,4$ года. Критерии включения: хроническая сердечная недостаточность (ХCH) III-IV функционального класса (ФК) по NYHA, фракция выброса левого желудочка (ФВ ЛЖ) <40\%, подтвержденный гистологически диагноз ВКМП или ДКМП, отсутствие признаков воспалительной реакции. Критерии невключения: наличие гемодинамически значимых стенозов на коронароангиографии (КАГ), нежелание пациента участвовать в исследовании, наличие онкопатологии.

С целью подбора пациентов с ДКМП и ВКМП, сопоставимых по состоянию внутрисердечной гемодинамики и достижения исходной гомогенности групп, применяли метод PSM (Propensity score matching). Пары 
пациентов были сформированы методом подбора с помощью поиска «ближайшего соседа» на основании таких ключевых показателей, как конечно-диастолический объем (КДО) ЛЖ, конечно-систолический объем (КСО) ЛЖ и давление в легочной артерии (ДЛА). Данный способ позволил сформировать 34 пары пациентов, распределенных в две группы: группа 1 - лица с ДКМП ( $\mathrm{n=32})$, группа 2 - пациенты с ВКМП ( $\mathrm{n=32}$ ).

Обследование пациентов включало: клинический осмотр, тест 6-минутной ходьбы, электрокардиограмму (ЭКГ) в 12 отведениях, трансторакальную эхокардиографию (ЭхоКГ) с использованием 2Dspeckletrackingstrain (продольная деформация миокарда левого желудочка), магнитно-резонансную томографию (МРТ) сердца с отсроченным контрастированием миокарда парамагнитным контрастным агентом гадолинием, клинико-лабораторные исследования (общий анализ крови, общий анализ мочи, биохимический анализ крови, концентрация С-реактивного белка (СРБ), концевого фрагмента мозгового натрийуретического пептида (NT-pro-BNP), уровень ST2, интерлейкина-6 (ИЛ-6), молекул межклеточной адгезии (ICAM) и сосудистой адгезии (VCAM)).

Локальную сократимость оценивали в сегментах миокарда ЛЖ. Посегментарно определяли время преконтрастной релаксации миокарда и объем экстрацеллюлярного матрикса методом МРТ, вольтаж униполярного и биполярного электродов при выполнении транскатетерного электромеханического картирования миокарда с использованием аппарата NOGA.

После первичного обследования всем пациентам назначена оптимальная медикаментозная терапия, которая проводилась согласно клиническому протоколу (приложение 4 к постановлению Министерства здравоохранения Республики Беларусь № 59 от 06.06.2017) и включала ингибиторы ангиотензин-превращающего фермента, бета-блокаторы, антагонисты рецепторов альдостерона, диуретики, при необходимости - сердечные гликозиды и антиаритмики III класса (амиодарон). Через 6 месяцев медикаментозной терапии проведено повторное обследование пациентов с выполнением ЭКГ, ЭхоКГ и теста 6-минутной ходьбы.

Статистическую обработку результатов выполняли с применением пакета программ Statistica 19.0 и IBM SPSS 25.0. Количественные переменные представлены в виде медианы (Ме), 25 и 75 перцентилей. Сравнение групп выполняли с использованием U-критерия Манна - Уитни. Взаимосвязь переменных оценивали с помощью коэффициента ранговой корреляции Спирмена $\left(r_{s}\right)$. Классифицирующую способность количественных показателей оценивали с помощью ROC-анализа. Индикатором диагностической ценности показателя служила площадь под ROCкривой (AUC - area under curve) с нижней границей 95\% ДИ не менее 0,60. Наилучшим порогом отсечения (cut-off) для тестируемой переменной являлось значение с оптимальным балансом чувствительности и специфичности. Величина критического уровня значимости (р) для всех критериев и тестов принималась равной 0,050.

\section{- РЕЗУЛЬТАТЫ И ОБСУЖДЕНИЕ}

Сравнение групп пациентов, сформированных методом подбора пары по верифицированным гистологически и клинически диагнозам, показало, что исходно группы 1 и 2 не различались по результатам теста 
6-минутной ходьбы. Медианное значение пройденной дистанции в группе пациентов с ДКМП составило 325 (300; 350) м, в группе пациентов с ВКМП - $322(295 ; 368)$ м ( $\mathrm{p}=0,734)$.

Отсутствовали статистически значимые межгрупповые различия по исходным параметрам внутрисердечной гемодинамики и по показателю продольной деформации миокарда ЛЖ, определяемым с помощью трансторакальной ЭхоКГ (табл. 1).

Анализ результатов лабораторных тестов показал, что группы исследования были сопоставимы по концентрации NT-proBNP $(p=0,089)$ и ST2 ( $=0,143)$ в крови (табл. 2). Не получено статистически значимых различий исследуемых групп по плазменному уровню маркеров воспаления и цитокинов: СРБ ( $p=0,524)$, ICAM $(p=0,700)$, VCAM $(p=0,149)$ и ИЛ-6 $(\mathrm{p}=0,387)$.

По данным электромеханического картирования пациенты с ВКМП и ДКМП не различались по вольтажу биполярного электрода (2,6 (2,0; $3,9)$ мВ и $3,0(2,0 ; 3,9)$ мВ соответственно, $\mathrm{p}=0,351)$. Но отмечена тенденция к более высоким значениям униполярного электрода в группе пациентов с ВКМП $(9,9(8,5 ; 11,2)$ мВ по сравнению с $8,8(7,5 ; 10,5)$ мВ при ДКМП, $\mathrm{p}=0,078)$.

Таблица 1

Эхокардиографические показатели у пациентов с кардиомиопатиями неишемической этиологии (Me (LQ; UQ))

Table 1

Echocardiographic parameters in patients with cardiomyopathies of non-ischemic etiology (Me (LQ; UQ))

\begin{tabular}{|l|l|l|l|}
\hline Показатель & ВКМП & ДКМП & P \\
\hline ФВ ЛЖ, \% & $28(27 ; 34)$ & $27(26 ; 32)$ & 0,241 \\
\hline $\begin{array}{l}\text { Передне-задний размер левого предсер- } \\
\text { дия, мм }\end{array}$ & $43(42 ; 46)$ & $45(43 ; 48)$ & 0,149 \\
\hline Конечно-диастолический размер ЛЖ, мм & $75(67 ; 78)$ & $74(70 ; 79)$ & 0,910 \\
\hline Конечно-систолический размер ЛЖ, мм & $64(60 ; 68)$ & $63(59 ; 69)$ & 0,880 \\
\hline КДО ЛЖ, мл & $245(196 ; 299)$ & $250(208 ; 272)$ & 0,762 \\
\hline КСО ЛЖ, мл & $172(142 ; 225)$ & $183(149 ; 200)$ & 0,850 \\
\hline Продольная деформация миокарда ЛЖ, \% & $-8(-15 ;-5)$ & $-6(-10 ;-5)$ & 0,752 \\
\hline ДЛА, мм рт. ст. & $38(30 ; 45)$ & $40(31 ; 46)$ & 0,191 \\
\hline
\end{tabular}

Таблица 2

Лабораторные показатели у пациентов с кардиомиопатиями неишемической этиологии (Me (LQ; UQ))

Table 2

Laboratory parameters in patients with cardiomyopathies of non-ischemic etiology (Me (LQ; UQ))

\begin{tabular}{|l|l|l|l|}
\hline Показатель & ВКМП & ДКМП & P \\
\hline Креатинин, мкмоль/л & $88(77 ; 100)$ & $86(75 ; 96)$ & 0,821 \\
\hline СРБ, мг/л & $2,0(0,8 ; 2,6)$ & $2,4(1,0 ; 2,8)$ & 0,524 \\
\hline NT-ргоВNР, пг/мл & $985(664 ; 2040)$ & $1129(736 ; 1945)$ & 0,089 \\
\hline ST2, пг/мл & $29,3(19,4 ; 37,1)$ & $30,6(21,1 ; 36,9)$ & 0,143 \\
\hline ICАМ, нг/мл & $676(514 ; 734)$ & $698(559 ; 767)$ & 0,700 \\
\hline VСАМ, нг/мл & $664(618 ; 933)$ & $760(652 ; 1098)$ & 0,149 \\
\hline ИЛ-6, пг/мл & $1,99(0,77 ; 7,10)$ & $2,06(0,33 ; 8,31)$ & 0,387 \\
\hline
\end{tabular}


При выполнении МРТ выявлено, что при первичном обследовании медианное значение времени преконтрастной релаксации миокарда ЛЖ было достоверно больше у пациентов с ВКМП $(1059$ (1026; 1098) мс по сравнению с 1022 (996; 1047) мс при ДКМП, р=0,002). При ВКМП выше была и медиана объема экстрацеллюлярного матрикса, отражающего степень фиброзных изменений миокарда: $32(28 ; 34) \%$ по сравнению с $27(25 ; 31) \%$ при ДКМП ( $=0,006)$. МРТ-показатели толщины миокарда в систолу и диастолу были меньше у пациентов с ВКМП (толщина миокарда в систолу 5,9 $(4,3 ; 7,2)$ мм по сравнению с $6,4(5,5 ; 8,0)$ мм при ДКМП $(p=0,039)$; толщина миокарда в диастолу $4,4(3,4 ; 5,5)$ мм по сравнению с $5,2(4,4 ; 6,4)$ мм при ДКМП $(p=0,002))$.

В целом по выборке пациентов с неишемической кардиомиопатией получены статистически значимые взаимосвязи показателей, ассоциированных с миокардиальным фиброзом. Объем экстрацеллюлярного матрикса миокарда ЛЖ умеренно коррелировал с фиброзом в биоптатах миокарда $\left(r_{s}=0,40, p=0,019\right)$ и вольтажем униполярного электрода $\left(r_{s}=-0,43, p=0,009\right)$. В свою очередь вольтаж униполярного электрода заметно коррелировал с гистологическим параметром фиброза $\left(r_{s}=-0,54\right.$, $\mathrm{p}<0,001)$. Следует отметить, что в гистологической картине кардиомиопатии наличие выраженного интерстициального фиброза, как правило, с развитием заместительного склероза, является основной отличительной чертой ВКМП. При этом, несмотря на отсутствие активного воспаления, для постмиокардитической кардиомиопатии характерно наличие рассеянных круглоклеточных воспалительных инфильтратов. Важно отметить, что в отличие от активного миокардита воспалительные инфильтраты не ассоциированы с повреждением миоцитов [6].

Для определения показателей, наиболее точно дифференцирующих ДКМП и ВКМП, был выполнен ROC-анализ с выбором пороговых значений, сбалансированных по чувствительности и специфичности классификации. Приемлемые значения AUC $(\geq 0,60)$ получены лишь для двух параметров MPT: времени преконтрастной релаксации (AUC -

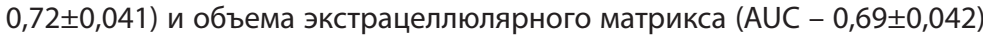
миокарда ЛЖ (см. рисунок).

Оптимальные пороговые значения для показателей, выделенных в результате анализа ROC-кривых, представлены в табл. 3. Наибольшие значения чувствительности и специфичности cut-off (>80\%) и наилучший их баланс достигнуты для объема экстрацеллюлярного матрикса.

Были определены границы 95\% ДИ количества сегментов, в которых значения данных параметров МРТ превышали cut-off. Для показателя времени преконтрастной релаксации получены следующие ДИ: при ВКМП - от 6 до 16, при ДКМП - от 5 до 8. Для показателя объема экстрацеллюлярного матрикса ДИ составили: при ВКМП - от 7 до 16, при ДКМП - от 2 до 8. Доверительные интервалы для ВКМП и ДКМП по времени преконтрастной релаксации и по показателю объема экстрацеллюлярного матрикса перекрывались в узком диапазоне (соответственно 6-8 сегментов и 7-8 сегментов), что дополнительно подтверждает значимость дискриминационной способности данных параметров МРТ при неишемической кардиомиопатии.

С учетом полученных данных и уже имеющихся клинических признаков, на основании которых может быть установлен диагноз у 


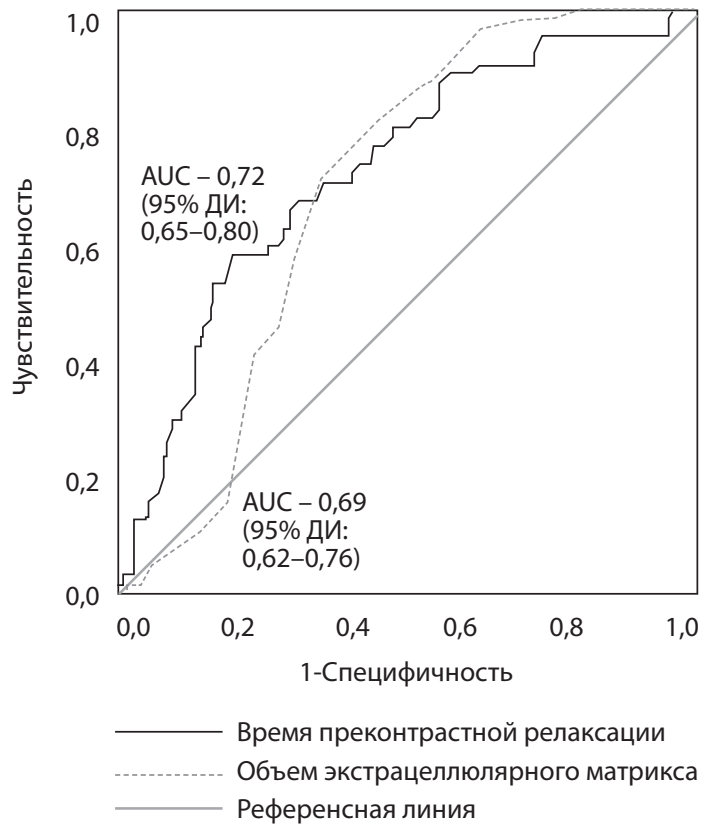

ROC-кривые показателей, эффективно классифицирующих случаи дилатационной и воспалительной кардиомиопатии

ROC-curves of parameters that effectively classify cases of dilated and inflammatory cardiomyopathy

пациентов с неишемической кардиомиопатией, был сформирован спектр критериев для дифференцированной диагностики идиопатической ДКМП и ВКМП с применением неинвазивных методов обследования (табл. 4). Известные основные и уточняющие диагностические критерии были дополнены следующими показателями МРТ, характерными для пациентов с ВКМП: время преконтрастной релаксации $\geq 1031$ мс и объем экстрацеллюлярного матрикса $\geq 28 \%$ не менее чем в 8 сегментах миокарда ЛЖ.

При повторном обследовании через 6 месяцев от начала оптимально подобранной медикаментозной терапии у пациентов обеих групп отмечалось увеличение пройденной дистанции в тесте 6-минутной ходьбы: у лиц с ДКМП до $458(395 ; 520)$ м (

\section{Таблица 3}

Пороговые значения (cut-off) показателей МРТ в сегментах миокарда пациентов с ДКМП и ПМКС с исходом в кардиомиопатию

Table 3

Cut-off MRI values in myocardial segments in patients with DCM and postmyocarditis cardiosclerosis resulting in cardiomyopathy

\begin{tabular}{|l|l|l|l|}
\hline \multirow{2}{*}{ Показатель } & \multicolumn{4}{|l|}{ Пороговое значение } \\
\cline { 2 - 4 } & сut-off & чувствительность & специфичность \\
\hline Время преконтрастной релаксации & 1031 мс & 77 & 79 \\
\hline $\begin{array}{l}\text { Объем экстрацеллюлярного ма- } \\
\text { трикса }\end{array}$ & $28 \%$ & 82 & 85 \\
\hline
\end{tabular}


Научные публикации. Оригинальные исследования

Таблица 4

Критерии дифференциальной диагностики неишемических кардиомиопатий

Table 4

Criteria for the differentiated diagnosis of non-ischemic cardiomyopathies

\begin{tabular}{|c|c|c|}
\hline $\begin{array}{l}\text { Диагностические кри- } \\
\text { терии }\end{array}$ & Воспалительная кардиомиопатия & Дилатационная кардиомиопатия \\
\hline Основные & $\begin{array}{ll}\text { - } & \text { дилатация полости ЛЖ; } \\
\text { - } & \text { фракция выброса ЛЖ <40\% }\end{array}$ & $\begin{array}{l}\text { - } \quad \text { дилатация полости ЛЖ; } \\
\text { - } \quad \text { фракция выброса ЛЖ <40\% }\end{array}$ \\
\hline Дополнительные & 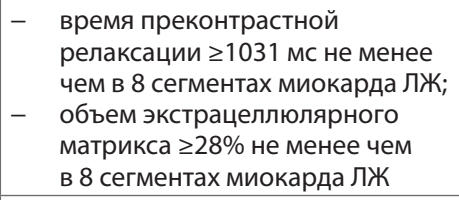 & $\begin{array}{ll}\text { - } & \text { время преконтрастной } \\
& \text { релаксации в сегментах } \\
\text { миокарда ЛЖ <1031 мс; } \\
\text { - } \\
\text { объем экстрацеллюлярного } \\
\text { матрикса в сегментах миокарда } \\
\text { ЛЖ <28\% }\end{array}$ \\
\hline Уточняющие & $\begin{array}{l}\text { - } \quad \text { наличие в анамнезе острых } \\
\text { и хронических вирусных и } \\
\text { бактериальных инфекционных } \\
\text { заболеваний }\end{array}$ & $\begin{array}{l}\text { - } \quad \text { отягощенный семейный анамнез } \\
\text { по кардиомиопатии и ранней, } \\
\text { внезапной смерти вследствие } \\
\text { кардиальных причин }\end{array}$ \\
\hline
\end{tabular}

$550(470 ; 608)$ м ( $p=0,012)$. Через 6 месяцев лечения группы 1 и 2 различались по значениям теста 6-минутной ходьбы ( $p=0,041)$.

По данным ЭхоКГ к 6-му месяцу лекарственной терапии у лиц с ВКМП фиксировалось улучшение внутрисердечной гемодинамики, о чем свидетельствовали уменьшение КДО ЛЖ (до $181(171 ; 222)$ мл, $\mathrm{p}=0,018)$ и КСО ЛЖ (до $127(104 ; 177)$ мл, $\mathrm{p}=0,017)$ и, как следствие, увеличение ФВ ЛЖ (до 32 (29; 37)\%, p=0,043). У пациентов с ДКМП, несмотря на их сопоставимость с группой ВКМП по стартовым (до начала терапии) эхокардиографическим показателям, не наблюдалось положительной динамики КДО ЛЖ $(228(185 ; 289)$ мл, $\mathrm{p}=0,121)$, КСО ЛЖ $(168(137 ; 237)$ мл, $p=0,162)$ и ФВ ЛЖ $(23(21 ; 38) \%, p=0,285)$ на фоне оптимальной медикаментозной терапии. Показатель продольной деформации миокарда Лж не претерпевал значимых изменений, что может быть обусловлено вариабельностью его значений в группах исследования.

В течение 6 месяцев оптимальной медикаментозной терапии у пациентов с ДКМП и ВКМП не выявлено статистически значимых изменений параметров МРТ: времени преконтрастной релаксации миокарда Лж (соответственно $p=0,123$ и $p=0,241$ ) и объема экстрацеллюлярного матрикса (соответственно $p=0,315$ и $p=0,752$ ). Следует отметить, что в ранее проведенном исследовании, посвященном эффективности терапии мезенхимальными стволовыми клетками (МСК) у пациентов с КМП неишемической этиологии, было показано снижение значений объема экстрацеллюлярного матрикса через 6 месяцев после интрамиокардиальной имплантации МСК у пациентов с ВКМП ( $p=0,007)$ [7]. При этом у лиц с ДКМП не наблюдалось положительной динамики данного показателя $(p=0,523)$. То есть изменения МРТ-показателя выраженности фиброза миокарда ЛЖ после введения МСК зависели от генеза КМП. По нашим данным, у пациентов с ВКМП, в отличие от лиц с ДКМП, терапия МСК превосходила оптимальную медикаментозную терапию и по положительному влиянию на локальную сократимость сегментов миокарда лж с акинезом [7]. 
- ВЫВОДЫ

1. Характерными особенностями впервые выявленной ВКМП являются более высокие, чем при ДКМП, количественные показатели фиброза миокарда ЛЖ (сегментарные значения времени преконтрастной релаксации $(p=0,002)$ и объема экстрацеллюлярного матрикса $(p=0,006))$ на фоне более низких показателей толщины миокарда Лж в систолу $(p=0,039)$ и диастолу $(p=0,043)$.

2. С применением ROC-анализа и анализа доверительных интервалов определены дополнительные критерии дифференциальной диагностики идиопатической ДКМП и ВКМП. Дополнительными диагностическими критериями ВКМП являются время преконтрастной релаксации $\geq 1031$ мс и объем экстрацеллюлярного матрикса $\geq 28 \%$ не менее чем в 8 сегментах миокарда Лж. Дополнительные критерии диагностики ДКМП: время преконтрастной релаксации <1031 мс и объем экстрацеллюлярного матрикса $<28 \%$.

3. Пациенты с гистологически подтвержденными диагнозами ДКМП и ВКМП, сопоставимые по показателям внутрисердечной гемодинамики, характеризуются различным ответом на оптимальную медикаментозную терапию. К 6-му месяцу лечения у лиц с ДКМП не наблюдается положительная динамика эхокардиографических параметров, у пациентов с ВКМП гемодинамика сердца улучшается, что выражается в уменьшении объемных показателей ЛЖ (КДО (p=0,018), КСО $(\mathrm{p}=0,017))$

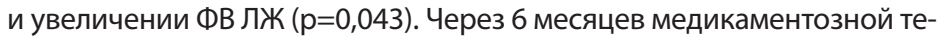
рапии пациенты с ВКМП преодолевают бо́льшую дистанцию в тесте 6-минутной ходьбы, чем лица с ДКМП ( $\mathrm{p}=0,041)$.

4. Разработанные дополнительные диагностические критерии с применением неинвазивного метода МРТ и особенности ответа пациентов с ДКМП и ВКМП на лечение являются предпосылкой для совершенствования дифференцированной тактики ведения пациентов с неишемическими кардиомиопатиями.

Конфликт интересов. Автор заявляет об отсутствии конфликта интересов.

Conflict of interest. The author declares no conflict of interest.

\section{ЛИТЕРАTУPA/REFERENCES}

Bakhchoyan M.R., Kosmacheva E.D., Slavinsky A.A., Verevkin A.A., Ponkina O.N., Chuprinenko L.M. (2017) Structural changes of myocardium at the expressed heart failure of non-coronarogenic etiology. Modern problems of science and education, no 5. Available at: http://www.science-education.ru/ru/article/view?id=26889 (date of access: 02.04.2021).

Amosova E.N. (1999) Cardiomyopathy. Kyiv: Kniga Plyus, 182 p. (in Russian)

Belenkov Yu.N. (2006) Chronic heart failure. Selected lectures on cardiology. M.: GEOTAR-Media, 432 p. (in Russian)

Metra M. (2007) Chronic heart failure: A position statement from the study group on advanced heart failure of the heart failure association of the European Society of

Cardiology. Eur. J. Heart Failure, vol. 9, pp. 684-694. (in English)
Kalenich O. (2002) Myocardial remodeling as the key factor in the development of circulatory insufficiency in cases of myocarditis. Cardiology, no 4, pp. $25-32$.

Alexandrova L.Z., Samsonov M.Yu., Osipov S.G., Masenko V.P. (1988) Immunological aspects of dilated cardiomyopathy. Clinical Medicine, no 6, pp. $48-51$.

Kurlyanskaya E.K. (2019) Effectiveness of Endomyocardial Administration of Mesenchymal Stem Cells in Patients with Cardiomyopathies of Non-Ischemic Etiology. Cardiology in Belarus, vol. 11, no 6, pp. 903-915.

Подана/Submitted: 09.09.2021

Принята/Accepted: 18.10 .2021

Контакты/Contacts: akurlianskaya@gmail.com 